\title{
SOBOLEV-TYPE APPROXIMATION RATES FOR DIVERGENCE-FREE AND CURL-FREE RBF INTERPOLANTS
}

\author{
EDWARD J. FUSELIER
}

\begin{abstract}
Recently, error estimates have been made available for divergencefree radial basis function (RBF) interpolants. However, these results are only valid for functions within the associated reproducing kernel Hilbert space (RKHS) of the matrix-valued RBF. Functions within the associated RKHS, also known as the "native space" of the RBF, can be characterized as vector fields having a specific smoothness, making the native space quite small. In this paper we develop Sobolev-type error estimates when the target function is less smooth than functions in the native space.
\end{abstract}

\section{INTRODUCTION AND PRELIMINARIES}

Radial basis functions (RBFs) have proven to be an effective tool in representing an unknown multivariate function by interpolation at scattered sites. They have been used in many applications, including medical imaging, computer animation and geometric design, neural networks, and the numerical solution of PDEs 1, 8, 9, 10. In many physical applications, it is desirable to have an approximant that exhibits certain physical characteristics, such as being divergence-free or curl-free. Narcowich and Ward introduced matrix-valued RBFs to help solve this problem 14. They constructed divergence-free vector-valued functions that interpolate data at scattered points. Also, a class of curl-free matrix-valued RBFs has recently been introduced 5 .

Constructing divergence-free and curl-free matrix-valued RBFs is fairly simple. If $\phi$ is a scalar-valued function consider

$$
\begin{aligned}
\Phi_{\text {div }} & :=\left(-\Delta I+\nabla \nabla^{T}\right) \phi, \\
\Phi_{\text {curl }} & :=-\nabla \nabla^{T} \phi .
\end{aligned}
$$

If $\phi$ is an RBF, then these functions can be used to produce divergence-free and curl-free interpolants, respectively. We note that these are not radial functions, but because they are usually generated by an RBF $\phi$, they are still commonly called "matrix-valued RBFs".

One constructs a divergence-free interpolant in the following way. Given a finite point set $X=\left\{x_{j}\right\}_{j=1}^{N} \subset \mathbb{R}^{n}$ and data $d_{j} \in \mathbb{R}^{n}$ associated with each $x_{j}$, we look

Received by the editor June 5, 2006 and, in revised form, April 8, 2007.

2000 Mathematics Subject Classification. Primary 41A63, 41A05; Secondary 41A30, 65D05.

The results are part of the author's dissertation written under the guidance of Francis Narcowich and Joe Ward at Texas A\&M University, College Station, Texas 77843. 
for coefficient vectors $\left\{c_{j}\right\}_{j=1}^{N} \subset \mathbb{R}^{n}$ so that

$$
\sum_{j=1}^{N} \Phi_{d i v}\left(x_{k}-x_{j}\right) c_{j}=d_{k} \quad \forall k=1, \ldots, N .
$$

This leads to the matrix equation

$$
A_{X, \Phi_{d i v}} c=d,
$$

where $c$ and $d$ are $n N \times 1$ vectors whose $j^{\text {th }} n$ components are given by $c_{j}$ and $d_{j}$, respectively. Also, $A_{X, \Phi_{d i v}}$ is an $n N \times n N$ matrix whose $(j, k)^{t h} n \times n$ block is given by $\Phi_{d i v}\left(x_{j}-x_{k}\right)$. This matrix is symmetric and positive definite, so (1) has a unique solution.

Error estimates for divergence-free RBF interpolation have recently been made available [11. However, these estimates only hold for a small class of functions within an RKHS of functions known as the "native space" of the matrix-valued RBF [5]. Native spaces are usually comprised of functions which are very smooth, so such error estimates are limited. Our main goal here is to show that when the Fourier transform of $\phi$ has algebraic decay, i.e.,

$$
c_{1}\left(1+\|\xi\|_{2}^{2}\right)^{-(\tau+1)} \leq \widehat{\phi}(\xi) \leq c_{2}\left(1+\|\xi\|_{2}^{2}\right)^{-(\tau+1)},
$$

then the approximation properties of matrix-valued RBFs extend to functions rougher than those in the native space. Finding estimates for functions outside the native space is sometimes referred to as "escaping" the native space.

Even for scalar-valued RBFs, this is a new development. The first "escape" was made by Narowich and Ward concerning functions on the $n$-sphere using spherical basis functions (SBFs), which are positive definite functions on the sphere [15. Results for RBFs on $\mathbb{R}^{n}$ followed 2, 16, 17. We refer the reader to 12 for a comprehensive overview of these findings. Due to applications to PDEs, it is desirable to obtain error estimates in terms of Sobolev norms. The above results address this partially, but they only have the appropriate norms on one side of the estimate. However, this issue has recently been completely resolved by Narcowich, Ward, and Wendland for a large class of RBFs, Wendland functions and thin plate splines in particular [18. Our strategy will be largely based on their approach, and the estimates we present will be of the form

$$
\left\|f-I_{X} f\right\|_{H^{k}(\Omega)} \leq h_{X, \Omega}^{\tau-k}\|f\|_{H^{\tau}(\Omega)},
$$

where $k \leq \tau$ is an integer, and $I_{X} f$ is the RBF interpolant to the target function $f$ on the point set $X$. Here $h_{X, \Omega}$ represents the mesh norm, given by

$$
h_{X, \Omega}:=\sup _{x \in \Omega} \inf _{x_{j} \in X}\left\|x-x_{j}\right\|_{2} .
$$

Another important quantity we will use in deriving our estimates is the separation radius of the point set $X$, given by

$$
q_{X}:=\inf _{j \neq k} \frac{1}{2}\left\|x_{j}-x_{k}\right\|_{2} .
$$

The paper is organized as follows. In the rest of this section, we introduce notation and state the necessary definitions. Next we discuss the approximation properties of divergence-free and curl-free band-limited functions. The results concerning band-limited functions are crucial in obtaining the main theorem. Finally, 
we present the main result of the paper, which is to show that interpolants arising from matrix-valued RBFs can effectively approximate functions that are more rough than those in the native space.

1.1. General notation. We will use $\|x\|_{2}$ to denote the standard Euclidean norm of $x \in \mathbb{R}^{n}$. If $A$ is a matrix, we will use $A^{+}$to denote its pseudoinverse. If $f$ is a matrix-valued function or distribution, we write $f^{*}$ for the conjugate transpose of $f$, i.e., $f^{*}=\bar{f}^{T}$. We define the ceiling function $\lceil x\rceil$ to be the function that returns the integer $k$ such that $k-1<x \leq k$. Also, we will work on domains that have smooth boundaries. We will say a bounded domain $\Omega \subset \mathbb{R}^{n}$ has $\mathcal{C}^{k, 1}$ boundary if the boundary is locally given by the graph of a function that is $k-1$ times continuously differentiable and has derivatives of order $k$ that are Lipschitz continuous.

Let $\Omega \subseteq \mathbb{R}^{n}$ and $f: \Omega \rightarrow \mathbb{R}$. We will say $f \in C^{k}(\Omega)$ if $f$ is $k$-times continuously differentiable. $L_{p}$ spaces are defined in the usual way: we say $f \in L_{p}(\Omega)$ if $\int_{\Omega}|f|^{p} d x$ is finite. In the case $\Omega=\mathbb{R}^{n}$, we define $C^{k}:=C^{k}\left(\mathbb{R}^{n}\right)$ and $L_{p}:=L_{p}\left(\mathbb{R}^{n}\right)$. Also, if $f$ is vector-valued, we say that $f \in C^{k}(\Omega)$ or $f \in L_{p}(\Omega)$ if each of its components are in $C^{k}(\Omega)$ or $L_{p}(\Omega)$, respectively. This should cause no confusion.

We will use the following convention for the Fourier transform of a function or tempered distribution:

$$
\widehat{f}(\xi):=\int_{\mathbb{R}^{n}} f(x) e^{-i x^{T} \xi} d \xi
$$

and let the inverse Fourier transform of a function or tempered distribution be defined by

$$
\check{f}(x):=\frac{1}{(2 \pi)^{n}} \int_{\mathbb{R}^{n}} f(\xi) e^{i \xi^{T} x} d \xi .
$$

If $f$ is a matrix-valued function, we will take $\widehat{f}$ to be the matrix of Fourier transforms of each component of $f$.

1.2. Sobolev spaces. The error estimates will deal with vector-valued functions whose components reside in the Sobolev spaces $W_{p}^{k}(\Omega)$, which are spaces that consist of all $f \in L^{p}(\Omega)$ that have distributional derivative $D^{\alpha} f$ in $L^{p}(\Omega)$ for all $\alpha$ with $|\alpha| \leq k$. The norms for these spaces are

$$
\|f\|_{W_{p}^{k}(\Omega)}:=\left(\sum_{|\alpha| \leq k}\left\|D^{\alpha} f\right\|_{L^{p}(\Omega)}^{p}\right)^{1 / p} .
$$

For the case $p=\infty$ we have

$$
\|f\|_{W_{\infty}^{k}(\Omega)}:=\max _{|\alpha| \leq k}\left\|D^{\alpha} f\right\|_{L^{\infty}(\Omega)}
$$

It is also possible to have Sobolev spaces of fractional order. Let $1 \leq p<\infty, k$ be a non-negative integer, and $0<t<1$. We define the Sobolev space $W_{p}^{k+t}(\Omega)$ to be all $u$ such that the following norm is finite:

$$
\|u\|_{W_{p}^{k+t}(\Omega)}:=\left(\|u\|_{W_{p}^{k}(\Omega)}^{p}+\sum_{|\alpha|=k} \int_{\Omega} \int_{\Omega} \frac{\left|D^{\alpha} u(x)-D^{\alpha} u(y)\right|^{p}}{\|x-y\|_{2}^{n+p t}} d x d y\right)^{1 / p} .
$$


The semi-norm for this space is given by

$$
|u|_{W_{p}^{k+t}(\Omega)}:=\left(\sum_{|\alpha|=k} \int_{\Omega} \int_{\Omega} \frac{\left|D^{\alpha} u(x)-D^{\alpha} u(y)\right|^{p}}{\|x-y\|_{2}^{n+p t}} d x d y\right)^{1 / p} .
$$

In the special case $p=2$, we define $H^{\tau}(\Omega):=W_{2}^{\tau}(\Omega)$. It is well-known that $H^{\tau}(\Omega)$ is a Hilbert space, and that in the case of $\Omega=\mathbb{R}^{n}$, we may use the Fourier transform to characterize $H^{\tau}\left(\mathbb{R}^{n}\right)$ :

$$
H^{\tau}\left(\mathbb{R}^{n}\right):=\left\{f \in L_{2}\left(\mathbb{R}^{n}\right): \widehat{f}(\cdot)\left(1+\|\cdot\|_{2}^{2}\right)^{\tau / 2} \in L_{2}\left(\mathbb{R}^{n}\right)\right\} .
$$

The inner product in $H^{\tau}\left(\mathbb{R}^{n}\right)$ is given by

$$
\langle g, f\rangle_{H^{\tau}\left(\mathbb{R}^{n}\right)}=\int_{\mathbb{R}^{n}}\left(1+\|\xi\|_{2}^{2}\right)^{\tau} \bar{f}(\xi) \widehat{g}(\xi) d \xi .
$$

For vector-valued Sobolev spaces, we use the following semi-norm and norm:

$$
|f|_{\left(W_{p}^{\tau}(\Omega)\right)^{n}}:=\left(\sum_{j=1}^{n}\left|f_{j}\right|_{W_{p}^{\tau}(\Omega)}^{p}\right)^{1 / p},\|f\|_{\left(W_{p}^{\tau}(\Omega)\right)^{n}}:=\left(\sum_{j=1}^{n}\left\|f_{j}\right\|_{W_{p}^{\tau}(\Omega)}^{p}\right)^{1 / p} .
$$

For $p=\infty$ we have:

$$
|f|_{\left(W_{\infty}^{\tau}(\Omega)\right)^{n}}:=\max _{1 \leq j \leq n}\left|f_{j}\right|_{W_{\infty}^{\tau}(\Omega)},\|f\|_{\left(W_{\infty}^{\tau}(\Omega)\right)^{n}}:=\max _{1 \leq j \leq n}\left\|f_{j}\right\|_{W_{\infty}^{\tau}(\Omega)} .
$$

With such norms $\left(H^{\tau}(\Omega)\right)^{n}$ is a Hilbert space, and in the special case $\Omega=\mathbb{R}^{n}$ the inner product can be defined by

$$
\langle g, f\rangle_{\left(H^{\tau}\left(\mathbb{R}^{n}\right)\right)^{n}}=\int_{\mathbb{R}^{n}}\left(1+\|\xi\|_{2}^{2}\right)^{\tau} \widehat{f}(\xi)^{*} \widehat{g}(\xi) d \xi .
$$

When the context is clear, we will use the notation $H^{\tau}(\Omega)=\left(H^{\tau}(\Omega)\right)^{n}$. This should cause no confusion.

We will also be interested in spaces that are divergence-free or curl-free. A function is divergence-free if and only if $\nabla \cdot f=0$. For $\tau \geq 0$, we define the space

$$
H_{\text {div }}^{\tau}(\Omega):=\left\{f \in H^{\tau}(\Omega): \nabla \cdot f=0\right\} .
$$

This is a closed subspace of $H^{\tau}(\Omega)$.

We would like to define a similar space for curl-free functions. In the case $n=2$, a function $f$ is curl-free if and only if $\partial f_{2} / \partial x-\partial f_{1} / \partial y=0$. When $n=3$, a function $f$ is curl-free if and only if $\nabla \times f=0$. We will use the sloppy notation $\nabla \times f$ to represent the curl of a vector field if $n=2$. Thus for $n=2$ or 3 we define

$$
H_{\text {curl }}^{\tau}(\Omega):=\left\{f \in H^{\tau}(\Omega): \nabla \times f=0\right\} .
$$

This is also a closed subspace of $H^{\tau}(\Omega)$. When $n>3$, there is no simple analogue for curl involving a nice differential operator. However, using differential forms and Poincaré's Lemma we see that a vector-valued function on a manifold has no rotation if and only if it is the differential of a scalar valued function. Therefore for general $n$ we will say a function $f \in H^{\tau}\left(\mathbb{R}^{n}\right)$ is curl-free on $\mathbb{R}^{n}$ if and only if there is a scalar-valued function in $H^{\tau+1}\left(\mathbb{R}^{n}\right) / \mathbb{R}$ such that $\nabla \phi=f$, and we define

$$
H_{\text {curl }}^{\tau}\left(\mathbb{R}^{n}\right):=\left\{f \in H^{\tau}\left(\mathbb{R}^{n}\right): \exists g \in H^{\tau+1}\left(\mathbb{R}^{n}\right) / \mathbb{R} \text { such that } \nabla g=f\right\} .
$$


1.3. Native spaces for matrix-valued kernels. In what follows we will need the notion of a "native space" for matrix-valued kernels. As in the scalar-valued theory, we will realize native spaces as reproducing kernel Hilbert spaces. We now define these for spaces of vector-valued functions.

Definition 1. Let $\mathcal{F}$ be a Hilbert space of vector-valued functions $f: \Omega \rightarrow \mathbb{R}^{n}$. A continuous $n \times n$ matrix-valued function $\Phi$ is called a reproducing kernel for $\mathcal{F}$ if for all $x \in \Omega$ and $c \in \mathbb{R}^{n}$ we have

1. $\Phi(\cdot-x) c \in \mathcal{F}$.

2. $c^{T} f(x)=(f, \Phi(\cdot-x) c)_{\mathcal{F}}$ for all $f \in \mathcal{F}$.

If $\mathcal{F}$ and $\Phi$ satisfy the above properties, we say $\mathcal{F}$ is the native space for the kernel $\Phi$ and we denote $\mathcal{F}$ by $\mathcal{N}_{\Phi}$.

In particular, we will be dealing with functions in the native spaces of the matrixvalued kernels $\Phi_{\text {div }}$ and $\Phi_{\text {curl }}$. These native spaces are equivalent to certain subspaces of Sobolev spaces, which we describe now. We define $\widetilde{H}^{\tau}$ to be the space of all functions $f \in\left(L_{2}\left(\mathbb{R}^{n}\right)\right)^{n}$ such that

$$
\|f\|_{\widetilde{H}^{\tau}}^{2}:=\int_{\mathbb{R}^{n}} \frac{\|\widehat{f}(\xi)\|_{2}^{2}}{\|\xi\|_{2}^{2}}\left(1+\|\xi\|_{2}^{2}\right)^{\tau+1} d \xi<\infty .
$$

Then $\widetilde{H}^{\tau}$ is a Hilbert space with inner product

$$
(f, g)_{\widetilde{H}^{\tau}}:=\int_{\mathbb{R}^{n}} \frac{\widehat{g}(\xi)^{*} \widehat{f}(\xi)}{\|\xi\|_{2}^{2}}\left(1+\|\xi\|_{2}^{2}\right)^{\tau+1} d \xi .
$$

It was shown in [5] that if $\phi$ satisfies (2), the native space of $\Phi_{d i v}$ is equivalent in norm to the subspace of divergence-free functions in $\widetilde{H}^{\tau}$, denoted by $\widetilde{H}_{d i v}^{\tau}$. Also, the native space for $\Phi_{\text {curl }}$ is equal to the corresponding space of curl-free functions, given by $\widetilde{H}_{c u r l}^{\tau}$. Also, it easy to see that if $f \in \widetilde{H}_{d i v}^{\tau}$, then $\|f\|_{\widetilde{H}^{\tau}}=\|f\|_{\widetilde{H}_{d i v}^{\tau}}$, and a similar equality holds for curl-free functions. Therefore when we measure functions in $\widetilde{H}_{\text {div }}^{\tau}$ or $\widetilde{H}_{\text {curl }}^{\tau}$ we will simply denote the norm by $\|\cdot\|_{\widetilde{H}^{\tau}}$. This should cause no confusion.

\section{BAND-LIMITED INTERPOLATION AND APPROXIMATION}

In scalar theory, escaping the native space relies heavily on using the approximation properties of band-limited functions, which are functions in $L_{2}$ whose Fourier transforms are compactly supported. Band-limited functions are analytic, and their smoothness puts them inside most native spaces. It turns out that such functions interpolate and approximate both functions in the native space and rougher functions, enabling one to essentially use a triangle inequality to escape the native space [18.

To make use of band-limited functions in the context of matrix-valued RBFs, we must ensure that they live within the native spaces of $\Phi_{d i v}$ and $\Phi_{c u r l}$. As seen in [5, functions in these native spaces must satisfy

$$
\int_{\mathbb{R}^{n}} \frac{\|\widehat{f}(\xi)\|^{2}}{\|\xi\|_{2}^{2}} d \xi<\infty
$$


Therefore we will work with the following band-limited spaces:

$$
\begin{aligned}
\mathcal{B}^{\sigma} & :=\left\{f \in\left(L_{2}\right)^{n}: \operatorname{supp}(\widehat{f}) \subset B(0, \sigma)\right\}, \\
\widetilde{\mathcal{B}}^{\sigma} & :=\left\{f \in \mathcal{B}^{\sigma}: \int_{\mathbb{R}^{n}} \frac{\|\widehat{f}(\xi)\|_{2}^{2}}{\|\xi\|_{2}^{2}} d \xi<\infty\right\}, \\
\widetilde{\mathcal{B}}_{\text {div }}^{\sigma} & :=\left\{f \in \widetilde{\mathcal{B}}^{\sigma}: \xi^{T} \widehat{f}(\xi)=0\right\}, \\
\widetilde{\mathcal{B}}_{\text {curl }}^{\sigma} & :=\left\{f \in \widetilde{\mathcal{B}}^{\sigma}: \widehat{f}(\xi)=\frac{\xi h(\xi)}{\|\xi\|_{2}}, h \in L_{2}\right\},
\end{aligned}
$$

where $B(0, \sigma)$ denotes a ball of radius $\sigma$.

2.1. Divergence-free and curl-free approximation. First we show that a divergence-free function in $\widetilde{H}^{t}$ can be approximated by a band-limited divergence-free function in $\widetilde{\mathcal{B}}_{d i v}^{\sigma}$. The proof is simple; one only has to "chop off" the Fourier transform of the function. The same proof works in the curl-free case, with the obvious modifications.

Lemma 1. Let $t \geq r \geq 0$. If $f \in \widetilde{H}^{t}$ is divergence-free, then for every $\sigma>0$ we have a function $g_{\sigma} \in \widetilde{\mathcal{B}}_{\text {div }}^{\sigma}$ with

$$
\left\|f-g_{\sigma}\right\|_{\widetilde{H}^{r}} \leq \sigma^{r-t}\|f\|_{\widetilde{H}^{t}} .
$$

Proof. To do this, we simply multiply the Fourier transform of $f$ by a cut-off function. Define $g_{\sigma}$ by $\widehat{g_{\sigma}}:=\widehat{f} \chi_{\sigma}$, where $\chi_{\sigma}$ is the characteristic function of the ball $B(0, \sigma)$. Since $\widehat{g}_{\sigma}$ is equal to a scalar function times $\widehat{f}$, then any relation that $\widehat{f}$ has with $\xi$ is inherited by $\widehat{g}_{\sigma}$. Thus $g_{\sigma}$ is divergence-free as long as $f$ is also. This also shows that if $f$ were curl-free, $g_{\sigma}$ would be curl free.

For the approximation, note that since $t \geq r$, for all $\|\xi\|_{2} \geq \sigma$ we have the inequality

$$
\left(1+\|\xi\|_{2}^{2}\right)^{r-t} \leq \sigma^{2(r-t)}
$$

This gives us

$$
\begin{aligned}
\left\|f-g_{\sigma}\right\|_{\widetilde{H}^{r}}^{2} & =\int_{\|\xi\| \geq \sigma} \frac{\|\widehat{f}(\xi)\|_{2}^{2}}{\|\xi\|_{2}^{2}}\left(1+\|\xi\|_{2}^{2}\right)^{r+1} d \xi \\
& =\int_{\|\xi\| \geq \sigma} \frac{\|\widehat{f}(\xi)\|_{2}^{2}}{\|\xi\|_{2}^{2}}\left(1+\|\xi\|_{2}^{2}\right)^{t+1}\left(1+\|\xi\|_{2}^{2}\right)^{r-t} d \xi \\
& \leq \sigma^{2(r-t)} \int_{\|\xi\| \geq \sigma} \frac{\|\widehat{f}(\xi)\|_{2}^{2}}{\|\xi\|_{2}^{2}}\left(1+\|\xi\|_{2}^{2}\right)^{t+1} d \xi \\
& \leq \sigma^{2(r-t)}\|f\|_{\widetilde{H}^{t}}^{2} .
\end{aligned}
$$

2.2. Divergence-free band-limited interpolation. Now it is our aim to show that we can simultaneously approximate and interpolate with divergence-free bandlimited functions. We will follow the approach used for the scalar-valued case in [18]. There it was shown that if one chooses $\sigma \sim 1 / q_{X}$, for every continuous function $f$ there exists a band-limited interpolant $f_{\sigma}$ whose Fourier transform is supported in $B(0, \sigma)$. We will prove something similar, and to do this we will need the following result from [16, Prop. 3.1]: 
Proposition 1. Let $\mathcal{Y}$ be a (possibly complex) Banach space, $\mathcal{V}$ be a subspace of $\mathcal{Y}$, and $Z^{*}$ be a finite dimensional subspace of $\mathcal{Y}^{*}$, the dual of $\mathcal{Y}$. If for every $z^{*} \in Z^{*}$ and some $\beta>1, \beta$ independent of $z^{*}$,

$$
\left\|z^{*}\right\|_{\mathcal{Y}^{*}} \leq \beta\left\|\left.z^{*}\right|_{\mathcal{V}}\right\|_{\mathcal{V}^{*}},
$$

then for $y \in \mathcal{Y}$ there exists $v \in \mathcal{V}$ such that $v$ interpolates $y$ on $Z^{*}$; that is, $z^{*}(y)=$ $z^{*}(v)$ for all $z^{*} \in Z^{*}$. In addition, $v$ approximates $y$ in the sense that $\|y-v\|_{\mathcal{Y}} \leq$ $(1+2 \beta) \operatorname{dist}(y, \mathcal{V})$.

We also require some results involving the space $\widetilde{H}_{d i v}^{\tau}$. This space can be characterized as a reproducing kernel Hilbert space for $\tau>n / 2$. The kernel $\widetilde{\mathcal{K}}_{d i v}^{\tau}$ is defined by its Fourier transform:

$$
\widehat{\widetilde{\mathcal{K}}_{\text {div }}^{\tau}}(\xi)=\left(\|\xi\|_{2}^{2} I-\xi \xi^{T}\right)\left(1+\|\xi\|_{2}^{2}\right)^{-(\tau+1)} .
$$

The inverse Fourier transform of $\left(1+\|\xi\|_{2}^{2}\right)^{-(\tau+1)}$ is given by

$$
\mathcal{K}^{\tau}:=c_{\tau}\|x\|_{2}^{\tau+1-n / 2} K_{\tau+1-n / 2}\left(\|x\|_{2}\right),
$$

where $K_{\nu}$ is the modified Bessel function of the second kind and $c_{\tau}$ is a constant 21. Theorem 6.13]. This gives us that $\widetilde{\mathcal{K}}_{\text {div }}^{\tau}$ can be written as

$$
\widetilde{\mathcal{K}}_{\text {div }}^{\tau}(x)=c_{\tau}\left(-\Delta I+\nabla \nabla^{T}\right)\|x\|_{2}^{\tau+1-n / 2} K_{\tau+1-n / 2}\left(\|x\|_{2}\right) .
$$

Now suppose that $X=\left\{x_{1}, \ldots, x_{N}\right\}$ is a set of distinct points from a bounded set $\Omega \subset \mathbb{R}^{n}$, and that $c_{1}, \ldots, c_{N}$ are vectors in $\mathbb{R}^{n}$. If $g:=\sum_{j=1}^{N} \widetilde{\mathcal{K}}_{d i v}^{\tau}\left(\cdot-x_{j}\right) c_{j}$, using the fact that $\widetilde{H}_{d i v}^{\tau}$ is a RKHS with kernel $\widetilde{\mathcal{K}}_{\text {div }}^{\tau}$ gives us

$$
\|g\|_{\widetilde{H}^{\tau}}^{2}=(2 \pi)^{n} \sum_{j, k} c_{j}^{*} \widetilde{\mathcal{K}}_{d i v}^{\tau}\left(x_{j}-x_{k}\right) c_{k} .
$$

As a result, we have that

$$
(2 \pi)^{n} \lambda_{X}\|c\|_{2}^{2} \leq\|g\|_{\widetilde{H}^{\tau}}^{2} \leq(2 \pi)^{n} \Lambda_{X}\|c\|_{2}^{2},
$$

where $\lambda_{X}$ and $\Lambda_{X}$ are the minimum and maximum eigenvalues of the $n N \times n N$ matrix $A_{X, \widetilde{\mathcal{K}}_{d i v}^{\tau}}$, and $c$ is the vector in $\mathbb{R}^{n N}$ whose $j^{\text {th }} n$-components are given by $c_{j}$.

We can get a lower bound to the minimum eigenvalues using the recent stability estimates given in [5]. The result is

$$
\lambda_{X} \geq c_{\tau, n} q_{X}^{2 \tau-n}
$$

where $q_{X}$ is the separation radius for $X$ and $c_{\tau, n}$ is a constant depending on its subscripts. To get upper bounds for $\Lambda_{X}$, we need to calculate the kernel explicitly. The function $K_{\nu}$ satisfies (see [20, page 79])

$$
\frac{d}{d z}\left(z^{-\nu} K_{\nu}(z)\right)=-z^{\nu} K_{\nu+1}(z) .
$$

Using this identity, one gets

$$
\widetilde{\mathcal{K}}_{\text {div }}^{\tau}=A(x) I+B(x)\left(-\|x\|_{2}^{2}+x x^{T}\right),
$$

where $A(x)$ and $B(x)$ are given by

$$
\begin{aligned}
& A(x):=c_{\tau} n\|x\|_{2}^{-(\nu+1)} K_{\nu+1}\left(\|x\|_{2}\right), \\
& B(x):=c_{\tau}\|x\|_{2}^{-(\nu+2)} K_{\nu+2}\left(\|x\|_{2}\right) .
\end{aligned}
$$


Here $\nu=\tau+1-n / 2$. From (8), we see that the eigenvalues of $\widetilde{\mathcal{K}}_{\text {div }}^{\tau}(x)$ are $A(x)-\|x\|_{2}^{2} B(x)$ with multiplicity $n-1$ and $A(x)$ with multiplicity 1 . This gives us that the modulus of the largest eigenvalue of the $n \times n$ matrix $\widetilde{\mathcal{K}}_{\text {div }}^{\tau}(x)$ is bounded by

$$
\Lambda_{\tilde{\mathcal{K}}_{\text {div }}^{\tau}}(x):=|A(x)|+\|x\|_{2}^{2}|B(x)| .
$$

We will also need the following estimate:

Proposition 2. The function $\|x\|_{2}^{-\nu} K_{\nu}\left(\|x\|_{2}\right)$ is positive, decreasing on $[0, \infty)$, and has the bound

$$
\|x\|_{2}^{-\nu} K_{\nu}\left(\|x\|_{2}\right) \leq \sqrt{2 \pi}\|x\|_{2}^{\nu-1 / 2} e^{-\|x\|_{2}+\frac{\nu^{2}}{2\|x\|_{2}}} .
$$

Proof. See Corollary 5.12 and Lemma 5.13 in [21.

Note that if $\|x\|_{2}>1$, we have:

$$
\begin{aligned}
\left|\Lambda_{\tilde{\mathcal{K}}_{\text {div }}^{\tau}}(x)\right| & \leq c_{\tau} n\|x\|_{2}^{-(\nu+1)} K_{\nu+1}\left(\|x\|_{2}\right)+c_{\tau}\|x\|_{2}^{2}\|x\|_{2}^{-(\nu+2)} K_{\nu+2}\left(\|x\|_{2}\right) \\
& \leq C_{\tau, n}\|x\|_{2}^{\nu+7 / 2} e^{-\|x\|_{2}}=: \Gamma(x)
\end{aligned}
$$

where $\nu=\tau+1-n / 2$. Furthermore, $\Gamma(x)$ will be decreasing with $\|x\|_{2}$ if $\|x\|_{2}>$ $\nu+7 / 2$. This can be shown by simple calculus.

Lemma 2. Let $g:=\sum_{j=1}^{N} \widetilde{\mathcal{K}}_{d i v}^{\tau}\left(\cdot-x_{j}\right) c_{j}$ and define $g_{\sigma}$ by $\widehat{g_{\sigma}}=\widehat{g} \chi_{\sigma}$, where $\chi_{\sigma}$ is the characteristic function of the ball $B(0, \sigma)$. Then, there exists a constant $\kappa>0$, which is independent of $X$ and the $c_{j}$ 's, such that for $\sigma=\kappa / q_{X}$ the following inequality holds:

$$
I_{\sigma}:=\left\|g-g_{\sigma}\right\|_{\widetilde{H}^{\tau}} \leq \frac{1}{2}\|g\|_{\widetilde{H}^{\tau}} .
$$

Proof. From the definition of $I_{\sigma}$ and the change of variables to $\xi=\sigma \xi$, we have

$$
\begin{aligned}
I_{\sigma}^{2} & =\int_{\|\xi\|_{2} \geq \sigma} \widehat{g}^{*}(\xi){\widehat{\mathcal{\mathcal { K }}_{d i v}}}^{+}(\xi) \widehat{g}(\xi) d \xi=\sigma^{n} \int_{\|\xi\|_{2} \geq 1} \widehat{g}^{*}(\sigma \xi) \widehat{\widetilde{\mathcal{K}}}_{\text {div }}+(\sigma \xi) \widehat{g}(\sigma \xi) d \xi \\
& =\sigma^{n} \int_{\|\xi\|_{2} \geq 1} \widehat{g}^{*}(\sigma \xi)\left(I-\frac{\xi \xi^{T}}{\|\xi\|_{2}^{2}}\right) \widehat{g}(\sigma \xi) \frac{\left(1+\sigma^{2}\|\xi\|_{2}^{2}\right)^{\tau+1}}{\|\sigma \xi\|_{2}^{2}} d \xi \\
& =\sigma^{n+2} \int_{\|\xi\|_{2} \geq 1}\left(\sum_{j} c_{j} e^{-i x_{j}^{T} \sigma \xi}\right)^{*} \frac{\left(\|\xi\|_{2}^{2} I-\xi \xi^{T}\right)}{\left(1+\sigma^{2}\|\xi\|_{2}^{2}\right)^{(\tau+1)}}\left(\sum_{k} c_{k} e^{-i x_{k}^{T} \sigma \xi}\right) d \xi
\end{aligned}
$$

Note that the matrix $\left(I\|\xi\|_{2}^{2}-\xi \xi^{T}\right)$ is a scaled projection, so the integrand is positive. Now since $\|\xi\|_{2} \geq 1$, we have the inequality

$$
\frac{1}{\left(1+\sigma^{2}\|\xi\|_{2}^{2}\right)^{\tau+1}} \leq \frac{2^{\tau+1}}{\sigma^{2 \tau+2}} \frac{1}{\left(1+\|\xi\|_{2}^{2}\right)^{\tau+1}}
$$


so that

$$
\begin{aligned}
I_{\sigma}^{2} & \leq 2^{\tau+1} \sigma^{n-2 \tau} \int_{\mathbb{R}^{n}}\left(\sum_{j} c_{j} e^{-i\left(\sigma x_{j}^{T}\right) \xi}\right)^{*} \frac{\left(\|\xi\|_{2}^{2} I-\xi \xi^{T}\right)}{\left(1+\|\xi\|_{2}^{2}\right)^{(\tau+1)}}\left(\sum_{k} c_{k} e^{-i\left(\sigma x_{k}^{T}\right) \xi}\right) d \xi \\
& =2^{\tau+1} \sigma^{n-2 \tau} \int_{\mathbb{R}^{n}}\left(\sum_{j} c_{j} e^{-i\left(\sigma x_{j}^{T}\right) \xi}\right)^{*} \widehat{\widetilde{\mathcal{K}}_{d i v}^{\tau}}(\xi)\left(\sum_{k} c_{k} e^{-i\left(\sigma x_{k}^{T}\right) \xi}\right) d \xi \\
& =(2 \pi)^{n} 2^{\tau+1} \sigma^{n-2 \tau} \sum_{j, k} c_{j}^{*} \widetilde{\mathcal{K}}_{d i v}^{\tau}\left(\sigma x_{j}-\sigma x_{k}\right) c_{k} \\
\text { (13) } & \leq(2 \pi)^{n} 2^{\tau+1} \sigma^{n-2 \tau} \Lambda_{\sigma X}\|c\|_{2}^{2} .
\end{aligned}
$$

In the last line of the inequality we have used the fact that $\widetilde{\mathcal{K}}_{d i v}^{\tau}$ is Hermitian, and the fact that $\widetilde{\mathcal{K}}_{d i v}^{\tau}$ is a reproducing kernel. From (6) and (7), we also have the estimate

so we obtain

$$
(2 \pi)^{n} c_{\tau, n} q_{X}^{2 \tau-n}\|c\|_{2}^{2} \leq\|g\|_{\widetilde{H}^{\tau}}^{2},
$$

$$
I_{\sigma}^{2} \leq 2^{\tau+1} c_{\tau, n}^{-1}\left(\sigma q_{X}\right)^{n-2 \tau} \Lambda_{\sigma X}\|g\|_{\widetilde{H}^{\tau}\left(\mathbb{R}^{n}\right)}^{2} .
$$

Now note that the set $\sigma X$ has separation distance $q_{\sigma X}=\sigma q_{X}$. This will enable us to choose $\sigma$ so that we get a uniform bound on $\Lambda_{\sigma X}$. Let $c$ be the unit eigenvector associated with $\Lambda_{\sigma X}$ and let $c_{j} \in \mathbb{R}^{n}$ be the $j^{t h} n$-components of $c$. Before we proceed, we note that in [13, Eq. 4.11] it was shown that one has the estimate

$$
\sum_{j \neq k} f\left(\left\|x_{j}-x_{k}\right\|_{2}\right) \leq 3^{n} \sum_{m=1}^{\infty} m^{n-1} \kappa_{f, m}
$$

where $f$ is a scalar valued function on $\mathbb{R}^{n}$ and $\kappa_{f, m}$ is given by

$$
\kappa_{f, m}:=\sup \left\{\left|f\left(\|x\|_{2}\right)\right|: m q_{X} \leq\|x\|_{2} \leq(m+1) q_{X}\right\} .
$$

Keeping this in mind, we choose $\sigma$ so that $\sigma q_{X} \geq \nu+7 / 2$ and use (11) to get:

$$
\begin{aligned}
\Lambda_{\sigma X} & =\sum_{j, k} c_{j}^{T} \widetilde{\mathcal{K}}_{d i v}^{\tau}\left(\sigma x_{j}-\sigma x_{k}\right) c_{k}=A(0)+\sum_{j \neq k} c_{j}^{T} \widetilde{\mathcal{K}}_{d i v}^{\tau}\left(\sigma x_{j}-\sigma x_{k}\right) c_{k} \\
& \leq A(0)+n \sum_{j \neq k}\left|\Lambda_{\tilde{\mathcal{K}}_{d i v}^{\tau}}\left(\sigma x_{j}-\sigma x_{k}\right)\right| \leq A(0)+n \sum_{j \neq k} \Gamma\left(\sigma x_{j}-\sigma x_{k}\right) \\
& \leq A(0)+n 3^{n} C_{\tau, n} \sum_{m=1}^{\infty} m^{n-1} \Gamma\left(m \sigma q_{X}\right) \\
& \leq A(0)+n 3^{n} C_{\tau, n} \sum_{m=1}^{\infty} m^{n-1} \Gamma(m):=C_{1, \tau, n} .
\end{aligned}
$$

Here we have used the fact that such a choice of $\sigma$ allows us to use (11), such a choice causes $\Gamma$ to be decreasing, and the above series is convergent since $\Gamma$ is rapidly decreasing. From this bound it follows that

$$
I_{\sigma}^{2} \leq 2^{\tau+1} C_{1, \tau, n} c_{\tau, n}^{-1}\left(\sigma q_{X}\right)^{n-2 \tau}\|g\|_{\widetilde{H}^{\tau}}^{2} .
$$

Now choose $\sigma q_{x}=\kappa$ so large that the factor multiplying $\|g\|_{\widetilde{H}^{\tau}}^{2}$ is less than $1 / 4$. Taking square roots gives us the result. 
Now we describe the scenario for proving the main result of this section. The result will follow from Proposition 1, and we will apply it to the following scheme:

$$
\mathcal{Y}=\widetilde{H}_{\text {div }}^{\tau}, \quad \mathcal{V}=\widetilde{\mathcal{B}}_{\text {div }}^{\sigma}, \quad Z^{*}=\operatorname{span}\left\{c^{T} \delta_{x_{j}}: c \in \mathbb{R}^{n}, x_{j} \in X\right\} .
$$

Theorem 1. Let $\tau, t \in \mathbb{R}$ such that $\tau>n / 2$ and $t>0$. Given $f \in \widetilde{H}_{\text {div }}^{\tau+t}$ and a point set $X \subset \mathbb{R}^{n}$ with separation distance $q_{X}$, there exists a function $f_{\sigma} \in \widetilde{\mathcal{B}}_{\text {div }}^{\sigma}$ such that

$$
\left.f\right|_{X}=\left.f_{\sigma}\right|_{X} \text { and }\left\|f-f_{\sigma}\right\|_{\widetilde{H}^{\tau}} \leq 5 \cdot \operatorname{dist}_{\widetilde{H}^{\tau}}\left(f, \widetilde{\mathcal{B}}_{\text {div }}^{\sigma}\right) \leq 5 \kappa^{-t} q_{X}^{t}\|f\|_{\widetilde{H}^{\tau+t}}
$$

with $\sigma=\kappa / q_{X}$, where $\kappa \geq 1$ depends on only $\tau$ and $n$.

Proof. The proof will follow from Proposition 1 once we establish the following. Given $z^{*} \in Z^{*}$, we need to show that

$$
\left\|z^{*}\right\|_{\left(\widetilde{H}_{d i v}^{\tau}\right)^{*}} \leq 2\left\|\left.z^{*}\right|_{\widetilde{\mathcal{B}}_{d i v}^{\sigma}}\right\|_{\left(\widetilde{H}_{d i v}^{\tau}\right)^{*}}
$$

Since $\widetilde{H}_{d i v}^{\tau}$ is a reproducing kernel Hilbert space with kernel $\widetilde{\mathcal{K}}_{d i v}$, then $\widetilde{\mathcal{K}}_{d i v}^{\tau}\left(x-x_{j}\right) c$ is the Riesz representer of the functional $c^{T} \delta_{x_{j}}$. It follows that if $z^{*}=\sum c_{j}^{T} \delta_{x_{j}}$ and $g=\sum \widetilde{\mathcal{K}}_{\text {div }}^{\tau}\left(\cdot-x_{j}\right) c_{j}$ we have $\left\|z^{*}\right\|_{\left(\widetilde{H}_{d i v}^{\tau}\right)^{*}}=\|g\|_{\widetilde{H}_{d i v}^{\tau}}$. Also, note that $\left\|\left.z^{*}\right|_{\widetilde{\mathcal{B}}_{\text {div }}^{\sigma}}\right\|_{\left(\widetilde{H}_{\text {div }}^{\tau}\right)^{*}}=\left\|g_{\sigma}\right\|_{\widetilde{H}_{\text {div }}^{\tau}}$, where $g_{\sigma}$ is defined by $\widehat{g_{\sigma}}=\widehat{g} \chi_{\sigma}$.

Now, by Lemma 2 for a sufficiently large $\kappa$ and all $\sigma=\kappa / q_{X}$ we have the estimate

$$
\begin{aligned}
\left\|g_{\sigma}\right\|_{\widetilde{H}^{\tau}} & \geq\|g\|_{\widetilde{H}^{\tau}}-\left\|g_{\sigma}-g\right\|_{\widetilde{H}^{\tau}} \\
& \geq\|g\|_{\widetilde{H}^{\tau}}-\frac{1}{2}\|g\|_{\widetilde{H}^{\tau}}=\frac{1}{2}\|g\|_{\widetilde{H}^{\tau}} .
\end{aligned}
$$

Thus the conditions of Proposition 1 are satisfied with $\beta=2$. The proposition tells us that for any divergence-free function $f \in \widetilde{H}_{\text {div }}^{\tau+t}\left(\mathbb{R}^{n}\right)$ there exists a divergencefree function $f_{\sigma} \in \widetilde{\mathcal{B}}_{\sigma}$, with $\sigma=\kappa / q_{X}$, such that $f_{\sigma}$ interpolates $f$ on $X$ and approximates it, in the sense that

$$
\left\|f-f_{\sigma}\right\|_{\widetilde{H}_{d i v}^{\tau}} \leq 5 \cdot \operatorname{dist}_{\widetilde{H}^{\tau}}\left(f, \widetilde{\mathcal{B}}_{d i v}^{\sigma}\right) .
$$

For the last inequality, we use Lemma 1 to get

$$
\operatorname{dist}_{\widetilde{H}^{\tau}}\left(f, \widetilde{\mathcal{B}}_{\text {div }}^{\sigma}\right)^{2} \leq \sigma^{-2 t}\|f\|_{\widetilde{H}^{\tau+t}}^{2} .
$$

Taking square roots and using the fact that $\sigma=\kappa / q_{X}$ gives us the result.

The estimates in Theorem 1 are exactly what one should expect based on the results of [18, Theorem 3.4]. There it was shown that a function $f$ in $H^{\tau+t}$ can be approximated by a band-limited interpolant with the estimate

$$
\left\|f-f_{\sigma}\right\|_{H^{\tau}} \leq 5 \cdot \operatorname{dist}_{H^{\tau}}\left(f, B_{\sigma}\right) \leq 5 \kappa^{-t} q_{X}^{t}\|f\|_{H^{\tau+t}} .
$$

In other words, if the error is being measured in a space that is $t$ degrees less smooth than the space the target function resides in, then the approximation rate should be given by $q_{X}^{t}$. Also, we would like to point out just how crucial the new stability estimates in [5] were in proving this result. In order to achieve the proper approximation rates, the power of $q_{X}$ from (7) had to exactly match that of $\sigma$ in (16), so that $\sigma$ could be chosen to "control" $q_{X}$. 
2.3. Curl-free band-limited interpolation. One can also interpolate and approximate a curl-free function with a band-limited curl-free function. The next result follows by using the same steps as in the proof of Theorem 1. replacing any divergence-free functions with the corresponding curl-free function. It also requires an estimate similar to that of Lemma 2 , which is easy to show by mimicking its proof.

Theorem 2. Let $\tau, t \in \mathbb{R}$ such that $\tau>n / 2$ and $t>0$. Given a function $f \in \widetilde{H}_{\text {curl }}^{\tau+t}$ and a point set $X \subset \mathbb{R}^{n}$ with separation distance $q_{X}$, there exists a curl-free function $f_{\sigma} \in \widetilde{\mathcal{B}}_{\text {curl }}^{\sigma}$ such that

$$
\left.f\right|_{X}=\left.f_{\sigma}\right|_{X} \text { and }\left\|f-f_{\sigma}\right\|_{\widetilde{H}^{\tau}} \leq 5 \cdot \operatorname{dist}_{\widetilde{H}^{\tau}}\left(f, \widetilde{\mathcal{B}}_{\text {curl }}^{\sigma}\right) \leq 5 \kappa^{-t} q_{X}^{t}\|f\|_{\widetilde{H}^{\tau+t}}
$$

with $\sigma=\kappa / q_{X}$, where $\kappa \geq 1$ depends on only $\tau$ and $n$.

\section{ERror estimates FOr FunCtions outside the NATIVE SPACE}

In this section we present the main result of the paper, which is to show that vector-valued $\mathrm{RBF}$ interpolants can approximate functions that are more rough than those in the native space. As mentioned earlier, the error estimates we give are in terms of the mesh norm. Another important value in the estimates is the mesh ratio, given by $\rho_{X, \Omega}:=h_{X, \Omega} / q_{X}$. In what follows, we will let $I_{X} f$ be the divergence-free RBF interpolant to $f$ on $X$ if $f$ is divergence-free, and the curl-free RBF interpolant to $f$ on $X$ if $f$ is curl-free. This should cause no confusion. Also, to be able to work on Sobolev spaces, we must assume that the Fourier transform of $\phi$ satisfies (2), which is the case when $\phi$ is a Sobolev spline or a Wendland function [21, Theorem 10.35].

3.1. Extensions of Sobolev spaces to the native space. To estimate error in the scalar-valued theory, one is able to start with a Sobolev function on a bounded domain and then extend the function continuously to $\mathbb{R}^{n}$ in a way that puts it inside of a native space, at least in the case where the native space is a Sobolev space. Once in the native space, best approximation properties of interpolants can be used to help estimate the error. It is our wish to do something similar here; that is, we want to extend divergence-free or curl-free Sobolev functions defined on a domain $\Omega$ to $\mathcal{N}_{\Phi_{\text {div }}}$ or $\mathcal{N}_{\Phi_{\text {curl }}}$. In the scalar-valued case, one has the advantage that the native spaces are Sobolev spaces, so well-known extension operators can be used. Native spaces for divergence-free and curl-free kernels are almost, but not quite, Sobolev spaces. However, when working on a bounded domain we will see that it is still possible to begin with a Sobolev function and extend it to the native space in a continuous manner. The ability to do this will depend upon the topology of $\Omega$. In what follows we will work on a bounded Lipschitz domain $\Omega \subset \mathbb{R}^{n}$. We will also assume $\Omega$ is simply connected, i.e., it has no "holes".

We will require our extension operators to extend functions continuously from $H^{\tau}(\Omega)$ to $\widetilde{H}^{\tau}\left(\mathbb{R}^{n}\right)$. Further, if a function is divergence-free or curl-free on $\Omega$, then the extensions should also be divergence-free or curl-free, respectively. We will be able to construct such operators for functions that can be written as the gradient or curl of a potential function. Most results involving vector potentials on Sobolev spaces are only proved in small dimensions, so in what follows $n=2$ or 3 . Once we obtain a potential, we will extend the original function by using Stein's extension operator on the potential. Recall that Stein's extension operator, which we denote 
by $\mathfrak{E}$, continuously extends any Sobolev function $f \in W_{p}^{\tau}(\Omega)$ to a function $\mathfrak{E} f \in$ $W_{p}^{\tau}\left(\mathbb{R}^{n}\right)[19$.

We will begin with the more simple case of curl-free functions. It is well-known that if $\Omega$ is a simply connected domain, $\nabla \times u=0$ for $u \in L_{2}(\Omega)$ if and only if there is a potential function $\phi \in H^{1}(\Omega)$ such that $u=\nabla \phi$. Note that when $u \in H^{\tau}(\Omega)$ we automatically get that $\phi \in H^{\tau+1}(\Omega)$. In order for the extension we construct to be continuous, we need an estimate of the form $\|\phi\|_{H^{\tau+1}(\Omega)} \leq C\|u\|_{H^{\tau}(\Omega)}$. Proving this is an easy application of the Closed Graph Theorem.

Lemma 3. Let $\tau \geq 0$ and let $\Omega \subset \mathbb{R}^{n}$ be a simply connected domain with Lipschitz boundary. There exists a continuous operator $T: H_{\text {curl }}^{\tau}(\Omega) \rightarrow H^{\tau+1}(\Omega)$ such that $u=\nabla(T u)$.

Proof. For each curl-free function $u$, we will let $T u$ be one of its potential functions. To ensure that $T$ is well-defined, let $T u$ be the potential of $u$ with minimum norm in $L_{2}(\Omega)$. It is clear that potentials of $u$ are unique up to a constant. Using this fact it is easy to show that if $\phi$ is any function such that $u=\nabla \phi$, then

$$
T u=\phi-\frac{1}{|\Omega|}\langle 1, \phi\rangle_{L_{2}(\Omega)},
$$

where $|\Omega|$ is the Lebesgue measure of $\Omega$. From this we get that $T$ is a welldefined linear operator. Also note that, in particular, taking $\phi=T u$ implies that $\langle 1, T u\rangle_{L_{2}(\Omega)}=0$.

Now we show that $T$ is a closed map. Suppose that $u_{n} \rightarrow u$ in $H^{\tau}(\Omega)$ and $T u_{n} \rightarrow \phi$ in $H^{\tau+1}(\Omega)$. We need to show that $T u=\phi$. This will follow if $\phi$ satisfies $u=\nabla \phi$ and $\langle 1, \phi\rangle_{L_{2}(\Omega)}=0$. We have

$$
\begin{aligned}
\|u-\nabla \phi\|_{H^{\tau}(\Omega)} & \leq\left\|u-u_{n}\right\|_{H^{\tau}(\Omega)}+\left\|\nabla\left(T u_{n}\right)-\nabla \phi\right\|_{H^{\tau}(\Omega)} \\
& \leq\left\|u-u_{n}\right\|_{H^{\tau}(\Omega)}+\left\|T u_{n}-\phi\right\|_{H^{\tau+1}(\Omega)} \rightarrow 0 .
\end{aligned}
$$

Similarly, we have

$$
\left|\langle 1, \phi\rangle_{L_{2}(\Omega)}\right|=\left|\left\langle 1, \phi-T u_{n}\right\rangle_{L_{2}(\Omega)}\right| \leq|\Omega||| T u_{n}-\phi \|_{L_{2}(\Omega)} \rightarrow 0 .
$$

Thus $T u=\phi$ and $T$ is closed. By the Closed Graph Theorem, $T$ is continuous, and we get the bound $\|T u\|_{H^{\tau+1}(\Omega)} \leq C\|u\|_{H^{\tau}(\Omega)}$.

With this result we are able to construct our extension operator. We extend $u$ by

$$
\widetilde{\mathfrak{E}}_{\text {curl }} u:=\nabla(\mathfrak{E} T u) \text {. }
$$

Note that $\widetilde{\mathfrak{E}}_{\text {curl }} u$ is automatically curl-free since it is the gradient of a scalar function. To show that $\widetilde{\mathfrak{E}}_{\text {curl }}: H_{\text {curl }}^{\tau}(\Omega) \rightarrow \widetilde{H}_{\text {curl }}^{\tau}\left(\mathbb{R}^{n}\right)$ is continuous, consider

$$
\begin{aligned}
\left\|\widetilde{\mathfrak{E}}_{\text {curl }} u\right\|_{\widetilde{H}_{\text {curl }}^{\tau}\left(\mathbb{R}^{n}\right)}^{2} & =\int_{\mathbb{R}^{n}} \frac{\left\|\widetilde{\mathfrak{E}_{\text {curl }} u}\right\|_{2}^{2}}{\|\xi\|_{2}^{2}}\left(1+\|\xi\|_{2}^{2}\right)^{\tau+1} d \xi \\
& =\int_{\mathbb{R}^{n}} \frac{\|\xi \widehat{\mathfrak{E}(T u)}\|_{2}^{2}}{\|\xi\|_{2}^{2}}\left(1+\|\xi\|_{2}^{2}\right)^{\tau+1} d \xi \\
& \leq \int_{\mathbb{R}^{n}}\|\widehat{\mathbb{E}(T u)}\|_{2}^{2}\left(1+\|\xi\|_{2}^{2}\right)^{\tau+1} d \xi=\|\mathfrak{E} T u\|_{H^{\tau+1}\left(\mathbb{R}^{n}\right)}^{2} \\
& \leq C\|T u\|_{H^{\tau+1}(\Omega)}^{2} \leq C\|u\|_{H^{\tau}(\Omega)}^{2} .
\end{aligned}
$$

This proves the following theorem. 
Theorem 3. Let $\tau \geq 0$ and let $\Omega \subset \mathbb{R}^{n}$ be a simply-connected bounded Lipschitz domain. Then there exists a continuous operator $\widetilde{\mathfrak{E}}_{\text {curl }}: H_{\text {curl }}^{\tau}(\Omega) \rightarrow \widetilde{H}_{\text {curl }}^{\tau}\left(\mathbb{R}^{n}\right)$ such that $\left.\widetilde{\mathfrak{E}}_{\text {curl }} u\right|_{\Omega}=u$ for all $u \in H_{\text {curl }}^{\tau}(\Omega)$.

Our strategy for the divergence-free case is the same: first we work on domains that allow for potential functions, construct a continuous operator $T$ that assigns a potential to each divergence-free function, and use Stein's operator $\mathfrak{E}$ to construct a continuous extension. We will only prove the results for $n=3$; the 2-dimensional case follows by a similar methodology. Also, we only state these results for integerordered Sobolev spaces (see Remark 1 below).

We will need several results concerning divergence-free functions. These are more or less well-known, but we will briefly list them with references for the curious reader. First, we get from [6, Theorem 3.4] that given a divergence-free vector field $u \in L_{2}(\Omega)$, we can find a vector potential $\phi \in H^{1}(\Omega)$ such that $\nabla \times \phi=u$. Furthermore, when $\Omega$ is simply connected, there is a unique vector potential such that

$$
\nabla \times \phi=u, \quad \nabla \cdot \phi=0, \quad \phi \cdot \vec{n}=0 \quad \text { on } \partial \Omega,
$$

where $\vec{n}$ is the normal vector to $\partial \Omega$. To determine the smoothness of the potential, we consider the following. Using [3, Corollary 7, Ch.9], we see that when $u \in H^{k}(\Omega)$ and the boundary of $\Omega$ is $\mathcal{C}^{\infty}$, then the potential $\phi$ is in $H^{k+1}(\Omega)$. A careful reading of the proof of this result, and the previous results in $[3$. on which it depends, shows that the regularity of the potential depends only on the regularity of the solutions of various elliptic boundary value problems, which can be applied to domains with rougher boundaries. According to [7, Theorem 2.5.1.1], the smoothness condition on the boundary can be relaxed from $\mathcal{C}^{\infty}$ to $\mathcal{C}^{k, 1}$. With these facts one can easily prove the following, again using the Closed Graph Theorem.

Lemma 4. Let $m \geq 0$ be an integer and let $\Omega$ be a simply-connected domain of $\mathbb{R}^{n}$ with $\mathcal{C}^{k, 1}$ boundary, where $k \geq m$ is an integer. Then there exists a continuous operator $T: H_{\text {div }}^{m}(\Omega) \rightarrow H^{m+1}(\Omega)$ such that $u=\nabla \times(T u)$.

Proof. For each divergence-free function $u$, we will let $T u \in H^{m+1}(\Omega)$ be the unique potential satisfying (17). From this we get that $T$ is well-defined, and we can easily check that it is linear.

As in the curl-free case, we show that $T$ is a closed map. Suppose that $u_{n} \rightarrow u$ in $H^{m}(\Omega)$ and $T u_{n} \rightarrow \phi$ in $H^{m+1}(\Omega)$. We need to show that $T u=\phi$. This will follow if $\phi$ satisfies (17). We have

$$
\begin{aligned}
\|u-\nabla \times \phi\|_{H^{m}(\Omega)} & \leq\left\|u-u_{n}\right\|_{H^{m}(\Omega)}+\left\|\nabla \times\left(T u_{n}\right)-\nabla \times \phi\right\|_{H^{m}(\Omega)} \\
& \leq\left\|u-u_{n}\right\|_{H^{m}(\Omega)}+C\left\|T u_{n}-\phi\right\|_{H^{m+1}(\Omega)} \rightarrow 0 .
\end{aligned}
$$

Similarly, we have

$$
\|\nabla \cdot \phi\|_{L_{2}(\Omega)}=\left\|\nabla \cdot \phi-\nabla \cdot T u_{n}\right\|_{L_{2}(\Omega)} \leq\left\|T u_{n}-\phi\right\|_{H^{m}(\Omega)} \rightarrow 0 .
$$

Also, the trace theorem gives us

$$
\|\phi \cdot \vec{n}\|_{L_{2}(\partial \Omega)}=\left\|\phi \cdot \vec{n}-T u_{n} \cdot \vec{n}\right\|_{L_{2}(\partial \Omega)} \leq C\left\|\phi-T u_{n}\right\|_{H^{1 / 2}(\Omega)} \rightarrow 0 .
$$

Thus $\phi$ satisfies (17) and $T$ is closed. By the Closed Graph Theorem, $T$ is continuous, and we get the bound $\|T u\|_{H^{m+1}(\Omega)} \leq C\|u\|_{H^{m}(\Omega)}$. 
With this result we are able to construct our divergence-free extension operator. We extend $u$ by

$$
\widetilde{\mathfrak{E}}_{\text {div }} u:=\nabla \times(\mathfrak{E} T u),
$$

where $\mathfrak{E} T u$ represents Stein's extension operating on each coordinate of the vector function $T u$. Note that $\widetilde{\mathfrak{E}}_{d i v} u$ is automatically divergence-free since it is the curl of a vector field. To show that $\widetilde{\mathfrak{E}}_{d i v}: H_{d i v}^{m}(\Omega) \rightarrow \widetilde{H}_{d i v}^{m}\left(\mathbb{R}^{n}\right)$ is continuous, consider

$$
\begin{aligned}
\left\|\widetilde{\mathfrak{E}}_{d i v} u\right\|_{\widetilde{H}_{d i v}^{m}\left(\mathbb{R}^{n}\right)}^{2} & =\int_{\mathbb{R}^{n}} \frac{\left\|\widehat{\widetilde{\mathfrak{E}}_{d i v} u}\right\|_{2}^{2}}{\|\xi\|_{2}^{2}}\left(1+\|\xi\|_{2}^{2}\right)^{m+1} d \xi \\
& =\int_{\mathbb{R}^{n}} \frac{\|\xi \times \widehat{\mathfrak{E}(T u)}\|_{2}^{2}}{\|\xi\|_{2}^{2}}\left(1+\|\xi\|_{2}^{2}\right)^{m+1} d \xi \\
& \leq C \int_{\mathbb{R}^{n}}\|\widehat{\mathbb{E}(T u)}\|_{2}^{2}\left(1+\|\xi\|_{2}^{2}\right)^{m+1} d \xi \\
& =C\|\mathfrak{E} T u\|_{H^{m+1}\left(\mathbb{R}^{n}\right)}^{2} \leq C\|T u\|_{H^{m+1}(\Omega)}^{2} \leq C\|u\|_{H^{m}(\Omega)}^{2} .
\end{aligned}
$$

This proves the following theorem for $n=2$ or 3 .

Theorem 4. Let $m \geq 0$ be an integer and let $\Omega \subset \mathbb{R}^{n}$ be a simply-connected domain with $\mathcal{C}^{k, 1}$ boundary, where $k \geq m$ is an integer. Then there exists a continuous operator $\widetilde{\mathfrak{E}}_{\text {div }}: H_{\text {div }}^{m}(\Omega) \rightarrow \widetilde{H}_{\text {div }}^{m}\left(\mathbb{R}^{n}\right)$ such that $\left.\widetilde{\mathfrak{E}}_{\text {div }} u\right|_{\Omega}=u$ for all $u \in H_{\text {div }}^{m}(\Omega)$.

Remark 1 . Note that in the divergence-free case we only showed that our extension operators were continuous for integer-ordered Sobolev spaces. The same is probably true for fractional-ordered Sobolev spaces, and this would follow immediately if one had interpolation results for divergence-free Sobolev spaces on bounded domains. In particular, we would need $H_{d i v}^{k+s}(\Omega)$ to be an interpolation space between $H_{d i v}^{k}(\Omega)$ and $H_{\text {div }}^{k+1}(\Omega)$, where $k \geq 0$ is an integer and $0<s<1$. When $\Omega=\mathbb{R}^{n}$, proving this is relatively straightforward. However, problems seem to arise when $\Omega \neq \mathbb{R}^{n}$, so we leave the fractional-ordered case for further future study.

3.2. Interpolation error estimates. With our extensions defined, we are now able to begin estimating interpolation approximation rates. In what follows we assume that $\Omega$ is a simply-connected domain with a $\mathcal{C}^{k, 1}$ boundary. Also, we require $\phi$ to satisfy (2) for some $\tau$. The proofs will only address the divergence-free case because they are essentially the same as in the curl-free case. We will begin by making use of a recent result from Narcowich, Ward, and Wendland concerning Sobolev estimates of functions with many zeros [17, Theorem 2.12]. In lieu of the remarks made in [18, Section 4], we will state the result in a slightly stronger form.

Proposition 3. Let $k$ be a positive integer, $0 \leq s<1,1 \leq p<\infty, 1 \leq q \leq \infty$, and let $\alpha$ be a multi-index satisfying $k>|\alpha|+n / p$, or $p=1$ and $k \geq|\alpha|+n$. Also, let $X \subset \Omega$ be a discrete set with mesh norm $h_{X, \Omega}$. Then there is a constant depending only on $\Omega$ such that if $h_{X, \Omega} \leq C_{\Omega}$ and if $u \in W_{p}^{k+s}(\Omega)$ satisfies $\left.u\right|_{X}=0$, then

$$
|u|_{W_{q}^{|\alpha|}(\Omega)} \leq C h_{X}^{k+s-|\alpha|-n(1 / p-1 / q)_{+}}|u|_{W_{p}^{k+s}(\Omega)},
$$

where $(x)_{+}=x$ is $x \geq 0$ and is 0 otherwise. Here the constant $C$ is independent of $h_{X, \Omega}$ and $u$.

One can use the relation between $p$ and $q$ norms to get the same result for $u \in\left(W_{p}^{k+s}(\Omega)\right)^{n}$. We will use this to prove our first error estimate, which bounds the error for a class of functions in the native space. 
Theorem 5. Let $k, s$, and $q$ be as in Proposition 3. Let $\tau=k+s$, and $m=\lceil\tau\rceil$. If $f \in H^{m}(\Omega)$ is divergence-free, we have

$$
\left\|f-I_{X} f\right\|_{W_{q}^{\beta}(\Omega)} \leq C h_{X, \Omega}^{\tau-\beta-n(1 / 2-1 / q)_{+}}\|f\|_{H^{m}(\Omega)},
$$

for all $\beta$ satisfying $0 \leq \beta \leq \tau$.

Proof. The remark after the previous proposition gives us that

$$
\left\|f-I_{X} f\right\|_{W_{q}^{\beta}(\Omega)} \leq C h_{X, \Omega}^{\tau-\beta-n(1 / 2-1 / q)_{+}}\left\|f-I_{X} f\right\|_{H^{\tau}(\Omega)} .
$$

Recall that in our case the native space is equivalent to $\widetilde{H}_{d i v}^{\tau}\left(\mathbb{R}^{n}\right)$. Now we continuously extend $f$ to $\widetilde{H}_{\text {div }}^{m}\left(\mathbb{R}^{n}\right)$ using $\widetilde{\mathfrak{E}}_{d i v}$ from Theorem 4 . Since $m \geq \tau$, we have that $\widetilde{\mathfrak{E}}_{\text {div }} f \in \widetilde{H}_{\text {div }}^{\tau}\left(\mathbb{R}^{n}\right)$. Once in $\widetilde{H}_{\text {div }}^{\tau}\left(\mathbb{R}^{n}\right)$ we can use the best approximation property of the interpolant in the native space to get

$$
\begin{aligned}
\left\|f-I_{X} f\right\|_{H^{\tau}(\Omega)} & \leq\left\|\widetilde{\mathfrak{E}}_{d i v} f-I_{X} f\right\|_{H^{\tau}\left(\mathbb{R}^{n}\right)} \leq C\left\|\widetilde{\mathfrak{E}}_{d i v} f-I_{X} f\right\|_{\widetilde{H}^{\tau}\left(\mathbb{R}^{n}\right)} \\
& \leq C\left\|\widetilde{\mathfrak{E}}_{d i v} f\right\|_{\widetilde{H}^{\tau}\left(\mathbb{R}^{n}\right)} \leq C\left\|\widetilde{\mathfrak{E}}_{d i v} f\right\|_{\widetilde{H}^{m}\left(\mathbb{R}^{n}\right)} \\
& \leq C\|f\|_{H^{m}(\Omega)} .
\end{aligned}
$$

Putting the above inequalities together finishes the proof.

We will also need to measure the error of the band-limited interpolant to the RBF interpolant of $f$. Note that in this case $f$ may not be in the native space.

Lemma 5. Let $\tau$ and $q$ be as in Theorem 5 , and let $\beta$ be an integer such that $\beta \leq \tau$ and $\beta>n / 2$. If $f \in H^{\beta}(\Omega)$ is divergence-free, let $f_{\sigma} \in \widetilde{\mathcal{B}}_{\sigma}$ be the interpolant to $\widetilde{\mathfrak{E}}_{\text {div }} f$ on $X$ from Theorem 11 with $t=0$. Then we have

$$
\left\|f_{\sigma}-I_{X} f_{\sigma}\right\|_{W_{q}^{\mu}(\Omega)} \leq C h_{X, \Omega}^{\beta-\mu-n(1 / 2-1 / q)_{+}} \rho_{X, \Omega}^{\beta-\tau}\|f\|_{H^{\beta}(\Omega)}
$$

for all $\mu$ satisfying $0 \leq \mu \leq \beta$.

Proof. Note that since $f_{\sigma}$ is band-limited and divergence-free, it is in $\widetilde{H}_{d i v}^{t}\left(\mathbb{R}^{n}\right)$ for all $t$. Since $f_{\sigma}-I_{X} f_{\sigma}$ is a function with zeroes on $X$, we get

$$
\left\|f_{\sigma}-I_{X} f_{\sigma}\right\|_{W_{q}^{\beta}(\Omega)} \leq C h_{X, \Omega}^{\tau-\beta-n(1 / 2-1 / q)_{+}}\left\|f_{\sigma}-I_{X} f_{\sigma}\right\|_{H^{\tau}(\Omega)} .
$$

We can estimate the right-hand side using the best approximation property of $I_{X} f_{\sigma}$ in $\widetilde{H}_{d i v}^{\tau}\left(\mathbb{R}^{n}\right)$ :

$$
\left\|f_{\sigma}-I_{X} f_{\sigma}\right\|_{H^{\tau}(\Omega)} \leq\left\|f_{\sigma}-I_{X} f_{\sigma}\right\|_{\widetilde{H}^{\tau}\left(\mathbb{R}^{n}\right)} \leq\left\|f_{\sigma}\right\|_{\widetilde{H}^{\tau}\left(\mathbb{R}^{n}\right)} .
$$

Since $\left(1+\|\xi\|_{2}^{2}\right)^{\tau+1} \leq \sigma^{\tau-\beta}\left(1+\|\xi\|_{2}^{2}\right)^{\beta+1}$ for $\|\xi\|_{2} \leq \sigma$, we have the Bernstein inequality

which implies that

$$
\left\|f_{\sigma}\right\|_{\widetilde{H}^{\tau}\left(\mathbb{R}^{n}\right)} \leq C q_{X}^{\beta-\tau}\left\|f_{\sigma}\right\|_{\widetilde{H}^{\beta}\left(\mathbb{R}^{n}\right)}
$$

$$
\left\|f_{\sigma}-I_{X} f_{\sigma}\right\|_{\widetilde{H}^{\tau}\left(\mathbb{R}^{n}\right)} \leq C h_{X, \Omega}^{\tau-\beta} q_{X}^{\beta-\tau}\left\|f_{\sigma}\right\|_{\widetilde{H}^{\beta}\left(\mathbb{R}^{n}\right)}=C \rho_{X, \Omega}^{\tau-\beta}\left\|f_{\sigma}\right\|_{\widetilde{H}^{\beta}\left(\mathbb{R}^{n}\right)} .
$$

All we have left to show is that $\left\|f_{\sigma}\right\|_{\widetilde{H}^{\beta}\left(\mathbb{R}^{n}\right)} \leq C\|f\|_{H^{\beta}(\Omega)}$. Using the approximation property of $f_{\sigma}$ and the continuity of $\widetilde{\mathfrak{E}}_{d i v}$ gives us:

$$
\begin{aligned}
\left\|f_{\sigma}\right\|_{\widetilde{H}^{\beta}\left(\mathbb{R}^{n}\right)} & \leq\left\|f_{\sigma}-\widetilde{\mathfrak{E}}_{d i v} f\right\|_{\widetilde{H}^{\beta}\left(\mathbb{R}^{n}\right)}+\left\|\widetilde{\mathfrak{E}}_{d i v} f\right\|_{\widetilde{H}^{\beta}\left(\mathbb{R}^{n}\right)} \\
& \leq C_{1}\left\|\widetilde{\mathfrak{E}}_{d i v} f\right\|_{\widetilde{H}^{\beta}\left(\mathbb{R}^{n}\right)}+C_{2}\|f\|_{H^{\beta}(\Omega)} \\
& \leq C\|f\|_{H^{\beta}(\Omega)} .
\end{aligned}
$$


The result thus follows.

Now we come to our main result, which is to estimate the RBF approximation error for divergence-free functions less smooth than those in the native space.

Theorem 6. Let $\tau$ and $q$ be as in Theorem [5] and let $\beta$ be a positive integer such that $\beta>n / 2$ and $\beta \leq \tau$. If $f \in H^{\beta}(\Omega)$ is a divergence-free function, then

$$
\left\|f-I_{X} f\right\|_{W_{q}^{\mu}(\Omega)} \leq C h_{X, \Omega}^{\beta-\mu-n(1 / 2-1 / q)_{+}} \rho_{X, \Omega}^{\tau-\beta}\|f\|_{H^{\beta}(\Omega)},
$$

where $\mu$ is any real number such that $0 \leq \mu \leq \beta$.

Proof. Using the fact that $f-I_{X} f$ is a function with many zeros, we get

$$
\left\|f-I_{X} f\right\|_{W_{q}^{\mu}(\Omega)} \leq C h_{X, \Omega}^{\beta-\mu-n(1 / 2-1 / q)_{+}}\left\|f-I_{X} f\right\|_{H^{\beta}(\Omega)} .
$$

The rest of the proof will be to estimate $\left\|f-I_{X} f\right\|_{H^{\beta}(\Omega)}$. We extend $f$ to $\widetilde{H}_{d i v}^{\beta}\left(\mathbb{R}^{n}\right)$ by $\widetilde{\mathfrak{E}}_{\text {div }} f$. According to Theorem[1] we may select a divergence-free function $f_{\sigma} \in \widetilde{\mathcal{B}}_{\sigma}$ with $\sigma=\kappa / q_{X}$ so that $\left.f_{\sigma}\right|_{X}=\left.\widetilde{\mathfrak{E}}_{\text {div }} f\right|_{X}$ and

$$
\left\|\widetilde{\mathfrak{E}}_{d i v} f-f_{\sigma}\right\|_{\widetilde{H}^{\beta}\left(\mathbb{R}^{n}\right)} \leq\left\|\widetilde{\mathfrak{E}}_{d i v} f\right\|_{\widetilde{H}^{\beta}\left(\mathbb{R}^{n}\right)} .
$$

Since $f_{\sigma}$ interpolates $f$ on $X$ implies that $I_{X} f=I_{X} f_{\sigma}$. This gives us

$$
\begin{aligned}
\left\|f-I_{X} f\right\|_{H^{\beta}(\Omega)} & \leq\left\|f-f_{\sigma}\right\|_{H^{\beta}(\Omega)}+\left\|f_{\sigma}-I_{X} f_{\sigma}\right\|_{H^{\beta}(\Omega)} \\
& \leq\left\|\widetilde{\mathfrak{E}}_{d i v} f-f_{\sigma}\right\|_{\widetilde{H}^{\beta}\left(\mathbb{R}^{n}\right)}+\left\|f_{\sigma}-I_{X} f_{\sigma}\right\|_{H^{\beta}(\Omega)} \\
& \leq C\left\|\widetilde{\mathfrak{E}}_{d i v} f\right\|_{\widetilde{H}^{\beta}\left(\mathbb{R}^{n}\right)}+\left\|f_{\sigma}-I_{X} f_{\sigma}\right\|_{H^{\beta}(\Omega)} \\
& \leq C\|f\|_{H^{\beta}(\Omega)}+\left\|f_{\sigma}-I_{X} f_{\sigma}\right\|_{H^{\beta}(\Omega)} .
\end{aligned}
$$

Now we use Lemma 5 to get

$$
\left\|f_{\sigma}-I_{X} f_{\sigma}\right\|_{H^{\beta}(\Omega)} \leq C \rho_{X, \Omega}^{\beta-\tau}\|f\|_{H^{\beta}(\Omega)} .
$$

Using the fact that $\rho_{X, \Omega} \geq 1$, we get

$$
\left\|f-I_{X} f\right\|_{H^{\beta}(\Omega)} \leq C \rho_{X, \Omega}^{\beta-\tau}\|f\|_{H^{\beta}(\Omega)} .
$$

We plug the above inequality into (19) to complete the proof.

Of course, there is an analogous theorem in the curl-free case. The proof follows from exactly the same arguments in this section. However, we can strengthen the result a bit by relaxing the smoothness of $\partial \Omega$. Here we only need to assume that the boundary is Lipschitz, while in Theorem 6 the boundary had to be $\mathcal{C}^{k, 1}$. Furthermore, we can prove that the result holds for fractional $\beta$.

Theorem 7. Let $\tau$ and $q$ be as in Theorem 5 and let $\beta$ be such that $\lfloor\beta\rfloor>n / 2$ and $\beta \leq \tau$. If $f \in H^{\beta}(\Omega)$ is a curl-free function, then

$$
\left\|f-I_{X} f\right\|_{W_{q}^{\mu}(\Omega)} \leq C h_{X, \Omega}^{\beta-\mu-n(1 / 2-1 / q)_{+}} \rho_{X, \Omega}^{\tau-\beta}\|f\|_{H^{\beta}(\Omega)},
$$

where $\mu$ is any real number such that $0 \leq \mu \leq \beta$. 


\section{REFERENCES}

1. R. K. Beatson, J. C. Carr, and W. R. Fright, Surface interpolation with radial basis functions for medical imaging, IEEE Trans. Med. Imaging 16 (1997), no. 1, 96-107.

2. Rob Brownlee and Will Light, Approximation orders for interpolation by surface splines to rough functions, IMA J. Numer. Anal. 24 (2004), no. 2, 179-192. MR2046173 (2005c:41013)

3. Robert Dautray and Jacques-Louis Lions, Mathematical analysis and numerical methods for science and technology. Vol. 3, Springer-Verlag, Berlin, 1990.

4. Edward J. Fuselier, Refined error estimates for matrix-valued radial basis functions, Ph.D. thesis, Texas A\&M University, 2006.

5. __ Improved stability estimates and a characterization of the native space for matrixvalued RBFs, Adv. Comput. Math. (2007) (to appear).

6. Vivette Girault and Pierre-Arnaud Raviart, Finite element methods for Navier-Stokes equations, Springer Series in Computational Mathematics, vol. 5, Springer-Verlag, Berlin, 1986, Theory and algorithms. MR851383 (88b:65129)

7. P. Grisvard, Elliptic problems in nonsmooth domains, Monographs and Studies in Mathematics, vol. 24, Pitman (Advanced Publishing Program), Boston, MA, 1985. MR775683 (86m:35044)

8. E. J. Kansa, Multiquadrics - a scattered data approximation scheme with applications to computational fluid-dynamics. I. Surface approximations and partial derivative estimates, Comput. Math. Appl. 19 (1990), no. 8-9, 127-145. MR1040157 (91b:65022)

9. — Multiquadrics - a scattered data approximation scheme with applications to computational fluid-dynamics. II. Solutions to parabolic, hyperbolic and elliptic partial differential equations, Comput. Math. Appl. 19 (1990), no. 8-9, 147-161. MR1040158(91b:65023)

10. N. Kojekine, Computer graphics and computer aided geometric design by means of compactly supported radial basis functions, Ph.D. thesis, Tokyo Institute of Technology, 2003.

11. Svenja Lowitzsch, Error estimates for matrix-valued radial basis function interpolation, J. Approx. Theory 137 (2005), no. 2, 238-249. MR2186949 (2006h:41042)

12. Francis J. Narcowich, Recent developments in error estimates for scattered-data interpolation via radial basis functions, Numer. Algorithms 39 (2005), no. 1-3, 307-315. MR2137758 (2006b:65017)

13. Francis J. Narcowich and Joseph D. Ward, Norms of inverses and condition numbers for matrices associated with scattered data, J. Approx. Theory 64 (1991), no. 1, 69-94. MR.1086096 (92b:65017)

14. functions, Math. Comp. 63 (1994), no. 208, 661-687. MR1254147 (95c:41014)

15. _ Scattered data interpolation on spheres: error estimates and locally supported basis functions, SIAM J. Math. Anal. 33 (2002), no. 6, 1393-1410. MR.1920637(2003j:41021)

16. Scattered-data interpolation on $\mathbb{R}^{n}$ : error estimates for radial basis and band-limited functions, SIAM J. Math. Anal. 36 (2004), no. 1, 284-300. MR2083863 (2005g:41011)

17. Francis J. Narcowich, Joseph D. Ward, and Holger Wendland, Sobolev bounds on functions with scattered zeros, with applications to radial basis function surface fitting, Math. Comp. 74 (2005), no. 250, 743-763. MR2114646 (2005k:41051)

18. Sobolev error estimates and a Bernstein inequality for scattered data interpolation via radial basis functions, Constr. Approx. 24 (2006), no. 2, 175-186. MR2239119 (2007g:41020)

19. Elias M. Stein, Singular integrals and differentiability properties of functions, Princeton Mathematical Series, No. 30, Princeton University Press, Princeton, N.J., 1970. MR0290095 (44:7280)

20. G. N. Watson, A treatise on the theory of Bessel functions, Cambridge Mathematical Library, Cambridge University Press, Cambridge, 1966. MR1349110 (96i:33010)

21. Holger Wendland, Scattered data approximation, Cambridge Monographs on Applied and Computational Mathematics, vol. 17, Cambridge University Press, Cambridge, 2005. MR:2131724 (2006i:41002)

Department of Mathematical Sciences, United States Military Academy, West Point, NEW YORK 10996

E-mail address: edward.fuselier@usma.edu 